\title{
Isolation of Cultivable Microorganisms from Polish Notes and Coins
}

\author{
MICHA£ KALITA, MARTA PALUSIŃSKA-SZYSZ, ANNA TURSKA-SZEWCZUK*, \\ SYLWIA WDOWIAK-WRÓBEL and TERESA URBANIK-SYPNIEWSKA
}

Department of Genetics and Microbiology, Institute of Microbiology and Biotechnology Maria Curie-Skłodowska University, Lublin, Poland

Submitted 23 August 2012, revised 6 May 2013, accepted 15 July 2013

\begin{abstract}
The potential role of currency in the spread of pathogenic microflora has been evaluated in many countries. In this study Polish paper notes and the coins in general circulation were assayed for the presence of cultivable bacteria and fungi. Bacterial isolates identification was based on cultural and biochemical characters and by comparison of the $16 \mathrm{~S}$ rRNA gene sequence. Fungal isolates were recognized with biochemical and morphological criteria. Coagulase-negative staphylococci, (43.6\% of the total bacterial count) including Staphylococcus saprophyticus, S. epidermidis, and S. hominis, and Enteroccus spp. (30.8\% of the total bacterial count), i.e. E. faecalis, E. faecium and E. durans, were the most numerous bacterial contamination. Penicillium spp., and Aspergillus spp. were the most frequently detected moulds whereas Candida spp. was the most frequent yeast isolated from currency. A visible dependence between the banknote denomination, the physical condition of paper currency, and the number of bacteria and fungi was found. The overall count of bacteria isolated from currency was thousand-fold higher than that of fungal isolates. The total amount of bacteria and fungi recovered from the coins was approximately 2.7 -fold lower than that isolated from the notes. In summary, the Polish currency notes were found to be contaminated mainly with commensal bacteria and fungi while the opportunistic pathogenic microorganisms Escherichia coli, Pseudomonas stutzeri and C. albicans were detected at a low frequency.
\end{abstract}

Ke y w o rds: Microbiological contamination; Polish currency; opportunistic pathogens transmission; 16S rRNA gene sequence analysis

\section{Introduction}

At the beginning of the seventies, banknotes and coins were reported as carriers of potentially pathogenic microorganisms (Jiang and Doyle, 1999; Khin et al., 1989). The risk of transmission of pathogenic microorganisms and diseases by currency was reported worldwide but most of these studies were performed in the tropical or sub-tropical regions of the world (Ahmed et al., 2010; Enemour et al., 2012; Yazah et al., 2012). Recent studies demonstrated that currency notes and coins were contaminated by bacteria, fungi, nematodes, and protozoa (Enemour et al., 2012; Kuria et al., 2009; Uneke and Ogbu, 2007) showed that a variety of bacterial species, mainly coagulase-negative staphylococci and spore-forming bacilli were identified in most of the note samples. The studies of Prasai et al. (2008) demonstrated the presence of heterotrophic aerobic bacteria on $98.4 \%$ of the total paper/polymer currency notes and coins tested. Besides bacteria, yeasts Candida spp., and Cryptococcus spp. (Kuria et al., 2009), and parasites - Ascaris lumbricoides and Enterobius vermicularis were isolated from banknotes in Africa (Uneke and Ogbu, 2007). These results indicate that currency notes may be a universal vehicle for transmission of pathogenic and potentially pathogenic microorganisms among persons either directly, through hand-to-hand contact, or indirectly, through water and food (Xu et al., 2005). The transfer of microorganisms from currency notes to humans depends on their number, ability to survive in the environment, and form of contact with currency. One critical factor for transmission of a microorganism from one person to another through everyday items is its ability to survive on the surface of the material. There are several factors influencing the persistence of microorganisms (Kramer et al., 2006).

The aim of this study was to investigate the frequency of the presence, and the nature of cultivable microbial contamination of currency notes and coins in circulation in Poland.

\section{Experimental}

\section{Materials and Methods}

Sample processing. A total of 150 samples of Polish currency banknotes in two denominations 10 and 20 Polish zloty (PLN) - 90 used and 10 new ones and

* Corresponding author: A. Turska-Szewczuk, Department of Genetics and Microbiology, Maria Curie-Skłodowska University, Akademicka 19, 20-033 Lublin, Poland; phone: (48 81) 537 5978; fax.: (48 81) 537 5959; e-mail: aturska@hektor.umcs.lublin.pl 
50 coins ( 1 and 2 Polish zloty) - 45 used and 5 new ones were collected in different cities from all over Poland. The samples were collected at random from various locations including grocery store, markets, flower shops, bus stations, restaurants, and banks. The banknotes and coins were placed into sterile plastic bags and immediately transported to the laboratory.

Qualitative and quantitative bacterial analysis. Each banknote and coin was aseptically placed into sterile tubes containing $10 \mathrm{ml}$ of trypticase soy broth (TSB) (BD, Heidelberg, Germany). The samples were vigorously shaken for 30 minutes to dislodge the microorganisms into the medium. Afterwards, the currency notes were aseptically removed with forceps. The undiluted samples and the serial dilutions (tenfold from $1 \times 10^{1}$ to $1 \times 10^{7}$ ) in sterile TSB were prepared and $0.1 \mathrm{ml}$ of the initial sample and each of the dilution was spread onto nutrient agar, blood agar, MacConkey, Chapman (Bio Merieux, Marcy, France) and Chromogenic UTI medium (Oxoid Ltd, Hampshire, England) plates, in triplicate. The plates were incubated aerobically for $24-48 \mathrm{~h}$ at $37^{\circ} \mathrm{C}$. The colonies that developed were counted and the total viable cells, referred to as CFU (colony forming units) per banknote and coin, were calculated. Bacteria were characterized on the basis of colony and cell morphology, Gram staining, and biochemical characters: the presence of coagulase and catalase, and utilization of various sugars using API tests (Bio Merieux, Marcy, France).

Qualitative and quantitative fungal analysis. Evaluation of the presence of fungi was performed by plating $0.1 \mathrm{ml}$ of overnight cultures from the banknotes and coins in TSB on Sabouraud's Dextrose agar (Biocorp, Warszawa, Poland) and incubation at $37^{\circ} \mathrm{C}$ for 48-72 $\mathrm{h}$ and then for 5 days at room temperature. The colonies were enumerated after 3 and 7 days of incubation. Mould fungi were identified, when possible, on the basis of cultural and microscopic characteristics. Yeast fungi were identified on the basis of colony and cell morphology and, where applicable, by biochemical tests. The cream-coloured, non-mucoid colonies resembling "Candida-like" yeast colonies were sub-cultured into Corn-Meal agar and examined in the germ-tube test.

Bacterial identification using 16S rRNA gene sequencing. Genomic DNA was isolated from representative strains using Genomic Mini kit (A\&A Biotechnology, Gdynia, Poland) according to the manufacturer's instructions. DNA concentration was determined using a spectrophotometer (SmartSpec 3000, Bio-Rad). 16S rDNA was amplified by PCR with fD1 and rD1 universal primers (Weisburg et al., 1991). The PCR cycling conditions were as follows: $3 \mathrm{~min}$ at $94^{\circ} \mathrm{C}$ of initial denaturation, $35 \times 45 \mathrm{sec}$ at $94^{\circ} \mathrm{C}, 1 \mathrm{~min}$ at $60^{\circ} \mathrm{C}, 2 \mathrm{~min}$ at $72^{\circ} \mathrm{C}$, and $7 \mathrm{~min}$ at $72^{\circ} \mathrm{C}$ of final elongation. Amplification was performed in a $25 \mu \mathrm{l}$ reaction mixture containing $1 \times$ REDTaq ReadyMix PCR mix (Sigma-Aldrich, St. Louis, MO), $0.1 \mu \mathrm{M}$ of each primer, and $100 \mathrm{ng}$ of genomic DNA template. PCR amplifications were carried out in a DNA thermal cycler (GeneAmp PCR System 2700; Applied Biosystems, Foster City, CA). PCR products running as a single band of the expected size of approximately 1500 bp were purified using Clean-Up kit (A\&A Biotechnology) according to the manufacturer's protocol. The 16S rDNA fragments were sequenced using fD1 and $\mathrm{rD} 1$ primers with the ABI Prism BigDye Terminator Cycle Sequencing Kit on the ABI Prism 3500 capillary sequencer (Applied Biosystems). All the sequences obtained were analyzed by the Bellerophon program (Huber et al., 2004) to remove chimeric 16S rRNA sequences. The $16 \mathrm{~S}$ rRNA sequences determined were queried against NCBI's sequence databases using on-line BLAST search (Madden et al., 1996). Subsequently, all sequences were aligned using the Clustal X 2.02 computer program (Thompson et al., 1997). The alignment was manually checked and corrected using BioEdit version 7 (Hall, 1999). A phylogenetic tree was generated by the neighbor joining (NJ) method using MEGA version 5 (Tamura et al., 2011). The genetic distances were calculated using the Kimura 2-parameter model (Kimura, 1980). A non-parametric bootstrap test (Felsenstein, 1995) was performed with 1000 replicates.

\section{Results}

Quantitative and qualitative characterization of bacteria and fungi isolated from currency notes and coins. The mean of the total viable bacteria counts recovered from the currency notes showed a value of $1.6 \times 10^{4} \mathrm{CFU}$ per a $\mathrm{N} 20$ note and $1.8 \times 10^{4} \mathrm{CFU}$ per a N10 note and of $6.5 \times 10^{3} \mathrm{CFU}$ per a coin. The mean values of the total fungi recovered from the notes and coins were 15.2 CFU and 5.5 CFU, respectively.

Out of the total samples of currency in circulation; $88 / 90=98 \%$ notes and $42 / 45=93 \%$ coins, were contaminated with bacteria. The level of bacterial and yeast contamination was related to the denomination of currency and was higher on the banknotes of lower denomination, i.e. $38 \%$ and $20 \%$, respectively, on the N10 notes in comparison to $33 \%$ and $10 \%$, respectively, on the N20 notes. The total number of mould fungi was similar on both denominations. The contamination was also related to the physical condition of the currency, since bacteria were isolated more frequently from dirty and mutilated notes. Our studies demonstrated that bacteria were isolated from all of the dirty or mutilated notes, whereas only $42 \%$ of used but clean notes were contaminated with bacteria. For comparison, neither bacteria nor fungi were isolated from new notes and coins. The results of qualitative and quantitative identification of bacteria and fungi are summa- 
Table I

The frequency of isolation of bacteria and fungi from Polish banknotes and coins in circulation

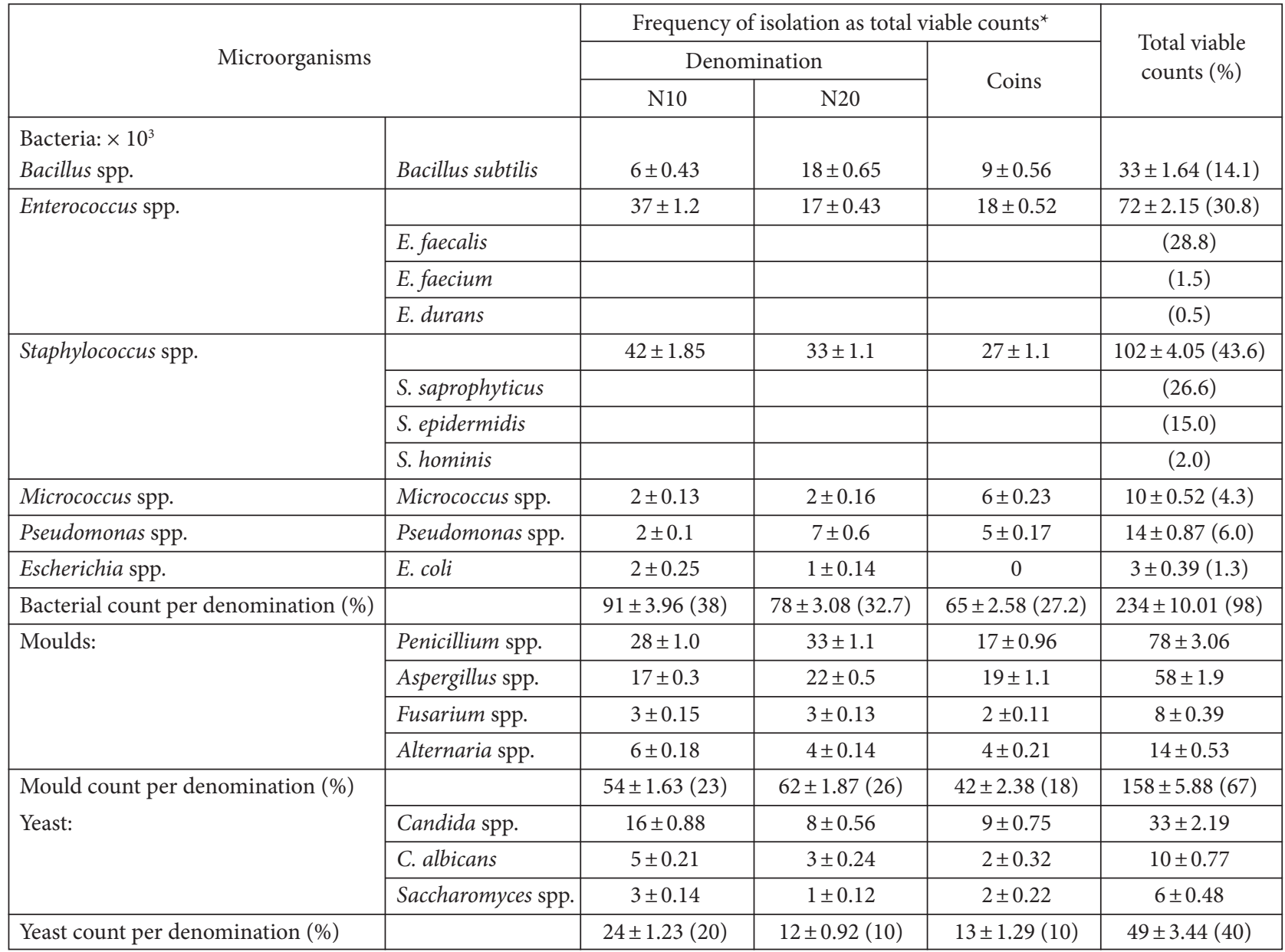

* Values are means of three replicates for each out of five notes

rized in Table I. The most numerous group of bacteria harvested from the banknotes and coins were catalasepositive, coagulase-negative bacteria classified to the genus Staphylococcus spp. (43.6\%) and Gram-positive catalase-negative cocci (35\%). Most likely, catalasenegative cocci belong to the genera Micrococcus and/ or Enterococcus, since only two species of Staphylococcus i.e. S. saccharolyticus and S. aureus subsp. anaerobius are not able to produce catalase (Kloos and Bannerman, 1999). The remaining isolates were identified as Grampositive bacilli (14.1\%) and Gram-negative rods (7.3\%). Gram-positive cocci represented $34.6 \%$ of the total bacterial isolates harvested from the N10 notes and 22.2\% of those collected from the N20 notes. Approximately half have been identified as coagulase-negative staphylococci, including S. saprophyticus, S. epidermidis, and S. hominis. Gram-positive, catalase-negative cocci isolated from $15.8 \%$ of $\mathrm{N} 10$ and $7.3 \%$ of the N20 currency notes and $7.7 \%$ of the coins were identified as Enterococus spp., i.e. E. faecalis, E. faecium and E. durans. Other catalase-negative cocci that accounted for $4.3 \%$ of the total bacterial count were recognized as belonging to the genus Micrococcus spp. Gram-positive spore forming bacilli, identified as Bacillus subtilis contrary to remaining Gram-positive genera, prevailed on the N20 notes; $10.6 \%$ of the total recovered bacterial count in comparison to $3.6 \%$ on the N10 notes. Gram-negative bacteria identified as Pseudomonas spp. prevailed on the notes of higher denomination, i.e. 3\% in comparison to $0.9 \%$. The other Gram-negative rods, isolated more frequently from the N10 notes, were identified as belonging to the genus Escherichia spp. Bacillus subtilis recovered from $93 \%$ of coin money represented approximately $14 \%$ of all the bacterial isolates. Gram-positive cocci outnumbered the isolates harvested from the used coins (78\%). A majority of isolates (42\%) were identified as coagulase-negative Staphylococcus spp. including S. epidermidis, S. saprophyticus and S. hominis. 37\% of the total bacterial isolates from the coins represented catalase-negative cocci, with most prevalent E. faecalis, E. faecium, and E. durans. In this group, bacteria recognized as Micrococcus spp. accounted for $9 \%$ of the total bacterial isolates from the coins. Nearly $8 \%$ of the total bacterial isolates from the coins accounted for aerobic rods of the genus Pseudomonas spp. Remarkably, no members of enteric bacteria were found. 
On the basis of cultural and microscopic characteristics, the following mould fungi Penicillium spp., Aspergillus spp., Fusarium spp., and Alternaria spp., were recovered with similar frequency from both denominations. $76 \%$ of the currency notes and $58 \%$ of the coins analyzed were contaminated by mould fungi. Penicillium spp. were the most predominant filamentous fungi (33\% of the total fungal isolates) followed by Aspergillus spp. (19\% of the total fungal isolates) that occurred on the currency notes and on the coins with equal number ( $9 \%$ of each genus). Other fungal contaminants were represented by Alternaria spp. and Fusarium spp., which constituted $4.8 \%$ and $2.9 \%$, respectively, of the isolates obtained from the banknotes. The yeast fungi were isolated from $46 \%$ of the currency notes and $34 \%$ from the coins. Among them, Candida (C. albicans and other than C. albicans) and Saccharomyces spp., 67\% and $12 \%$ respectively, were identified.

Bacterial identification using 16S rRNA gene sequencing and phylogenetic analysis of bacteria. The 16S rRNA gene sequencing reactions generated 1384-1487 bp long sequences that are available under the following accession numbers: JF923453-JF923465. All sequences obtained in this study were compared to sequence database in order to reveal their relationships to known bacterial genera. This approach allowed the identification of all DNA sequences of the resulting products. Comparison of DNA sequences of the PCR fragments obtained with $16 \mathrm{~S}$ rDNA bacterial sequences from the GenBank database demonstrated that the bacterial strains isolated from the banknotes and the coins belong to the genera that fit to the recognized

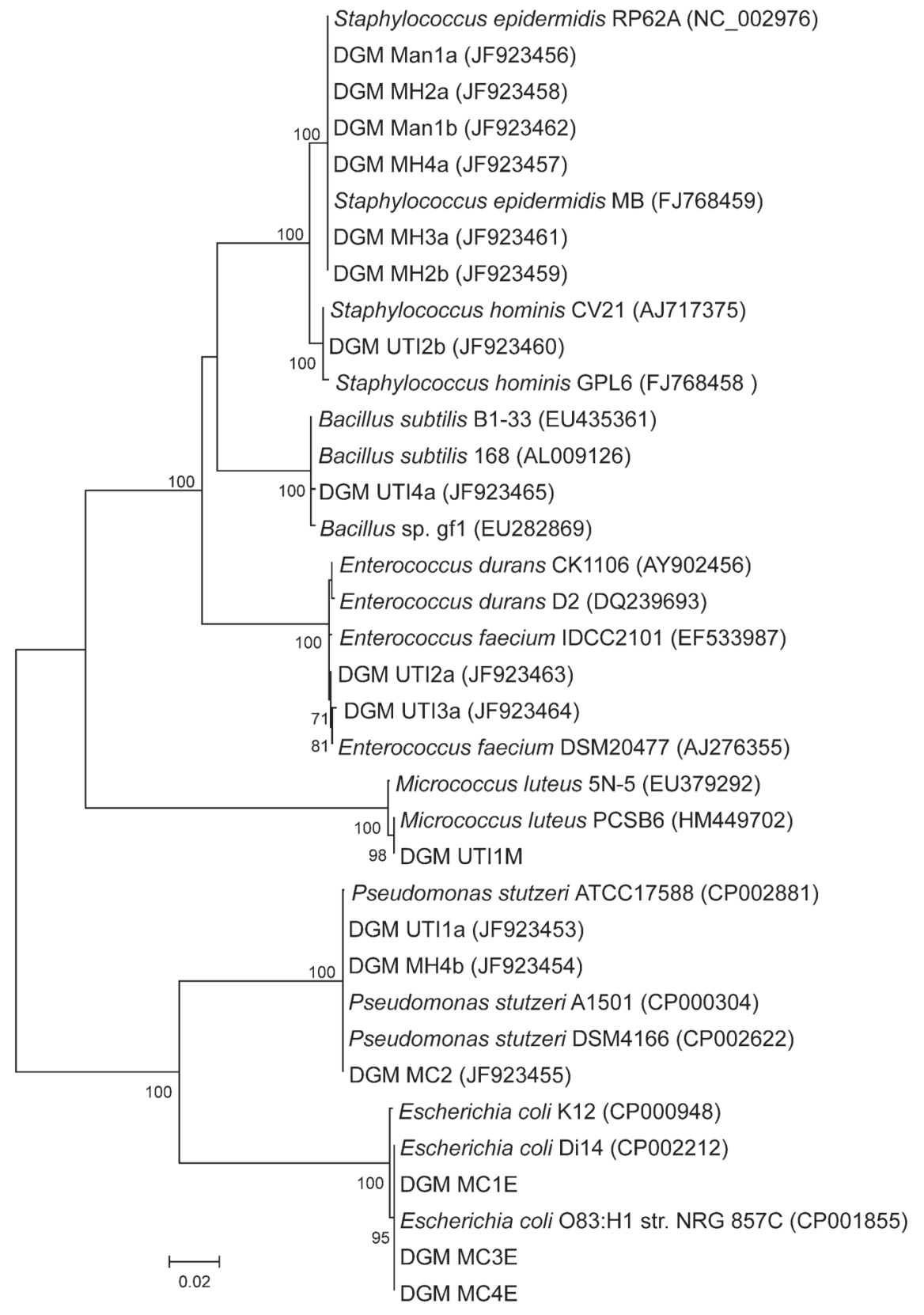

Fig. 1. Neighbor-joining phylogenetic tree based on the 1500-bp 16S rRNA gene sequences of strains isolated from Polish currency and reference bacterial species.

Bootstrap values $>70 \%$ are given at the branching points. The scale bar indicates the number of substitutions per site. 
phenotypic profiles i.e., Staphylococcus, Enterococcus, Micrococcus, Bacillus, Pseudomonas and Escherichia (Fig. 1). The nucleotide sequence alignments of the 16S rRNA gene of strains DGM Man1a, DGM MH2a, DGM Man1b, DGM MH4a, DGM MH3a, DGM MH2b identified as Staphylococcus shared $98-100 \%$ of identity with the sequences located in the databases. The topology and bootstrap values of the phylogenetic tree indicated that the strains from the genus Staphylococcus formed two coherent groups together with the strains S. epidermidis and S. hominis with $99-100 \%$ bootstrap support (Fig. 1). The bootstrap values allowed unambiguous assignment of the strains DGM UTI2a and DGM UTI3a to the genus Enterococcus.

Based on the phylogenetic analysis, the strains DGM UTI1a, DGM MC2, DGM MH4b formed a common cluster with $P$. stutzeri supported by a bootstrap value of $100 \%$. The other strains displayed essential similarity to Bacillus spp., isolate DGM UTI4a (the sequence similarity value of $98-100 \%)$, Micrococcus spp. strain DGM UTI1M (99-100\% sequence similarity) and Escherichia spp. strains DGM MC1E, DGM MC3E, DGM MC4E (100\% sequence similarity). All these strains belong to the phylogroups comprising the validly described species.

\section{Discussion}

In the present study, the identification and enumeration of bacteria and fungi isolated from the currency notes and coins collected at random from various places in Poland is described. The results indicated that $98 \%$ of the paper notes were contaminated with bacteria, $76 \%$ with mould fungi, and $34 \%$ with yeasts. A similar pattern of contamination was associated with the coins, in which the frequency of isolation of bacteria, mould, and yeasts was of $93 \%, 58 \%$ and $46 \%$, respectively. The control samples of new notes and coins tested under the same procedure indicated that they were free of microorganisms. Generally, the coins were less contaminated than the paper notes, and the banknotes of lower denomination were more often colonized than those of the higher one. This can be expected, as the coins are more durable than the paper currency and the exchange rate of lower denomination notes is higher. The average total bacterial count was $1.7 \times 10^{4}$ and fungal 15 CFU per note. For comparison, $6.5 \times 10^{3}$ of total bacterial count and $4.2 \mathrm{CFU}$ of total fungi per coin were obtained. This result could be explained by antibacterial effects of the metals the coins are made from. Polish coins, as most coins, are made from copper alloy; the bicolored two- and five-zloty coins are composed of different copper alloys (http://www.nbp.pl/homen. aspx?f=/en/banknoty/monety.htm). Metallic copper surfaces were demonstrated to inactivate microbes efficiently upon contact, especially when exposed to dry surfaces (Santo et al., 2010).

The results of cultural, morphological, biochemical and phylogenetic studies indicated the predominant currency-associated microorganisms belong to the bacterial genera Staphylococcus spp. (43.6\% of the total bacterial count), Enterococcus spp. (30.8\%), Bacillus subtilis (14\%), Micrococcus spp. (4\%), Pseudomonas spp. (6\%), and Escherichia coli (1.3\%), whereas mould fungi and yeasts were thousand-fold less frequently recovered. Out of 207 fungal isolates, Penicillium and Aspergillus filamentous fungi and yeasts identified as Candida spp., and C. albicans were most commonly found. Although Candida is a compound of normal human microflora it is also a common cause of opportunistic infections in immunocompromised persons (Patel et al., 2006).

The obtained data are in contrast with the results of studies performed on currency from other countries indicating the potential of such currencies for possible disease spread in the human communities (Khin et al., 1989; Kuria et al., 2009; Yazah et al., 2012). To reduce the amount of microbial contamination of currency in countries like Japan heat-sterilization of money is applied or paper money is being replaced by polymer banknotes (Prasai et al., 2008).

Staphylococcus saprophyticus appeared to be most habitual contamination of money in Poland $(26.6 \%$ of total bacterial counts). The presence of staphylococci is usually indicative of contamination from the skin, mouth, or nose. However, some strains of Staphylococcus, including coagulase-negative, can cause a number of diseases (Santo et al., 2010). Enterococcus species that were detected on the surface of the coins and paper money (30.8\% of the total bacterial isolates) are a part of the normal intestinal flora of humans and animals. Widely distributed in nature, B. subtilis was one of the most common bacterial contaminations found on both the coin surface and paper money ( $14 \%$ of the total bacterial count). Only $1 \%$ of the paper currency examined was found to be contaminated with E. coli strains. The presence of enteric bacteria e.g., coliforms and E. coli has been widely accepted as an indicator of fecal contamination. Such information, however valuable, should not be interpreted as indicating with certainty that fecal contamination has occurred (Jiang and Doyle, 1999).

About $6 \%$ of the money samples were contaminated with Pseudomonas stutzeri. In the case of this species, the problem of the accuracy of identification is related to its existence as "complexes" (phenospecies), as described by (Janda and Abbott, 2007). P. stutzeri is found in the environment, but is not a member of normal human flora and is rarely identified as a causative agent of human infections (Lalucat et al., 2006). Data on the persistence of nosocomial pathogens on inanimate 
surfaces are summarized in the review paper of Kramer et al. (2006). The most common nosocomial pathogens Enterococcus spp. (including VRE), S. aureus (including MRSA), Streptococcus pyogenes, Acinetobacter spp., E. coli, Klebsiella spp., P. aeruginosa, and Candida albicans may survive for months on dry surfaces.

Many studies on currency-associated microorganisms were largely based on phenotypic analysis (Ahmed et al., 2010; Prasai et al., 2008). 16S rRNA gene sequencing was used in identification of bacterial strains isolated from copper alloy coins (Santo et al., 2010; Xu et al., 2005). It was shown that Gram-positive bacilli and staphylococci predominate among bacteria found on the surface of copper coins (Santo et al., 2010). The unambiguous identification of the strains to the genus level using $16 \mathrm{~S}$ rDNA sequencing has demonstrated that Enterococcus spp. and Staphylococcus spp. are also prevailing bacteria found on Polish coins. This approach discriminates bacterial strains more accurately than it is possible with phenotypic analysis, allowing identification of strains that are poorly culturable or do not exhibit distinguishable phenotypic traits (Clarridge, 2004; Janda and Abbott, 2007).

Conclusions. This study was performed with the aim to isolate and identify cultivable bacteria and fungi from Polish notes and coins in circulation. The prevalence of commensal bacteria i.e. coagulase-negative Staphylococcus spp. and Enterococcus spp. (74.4\% of the total bacterial count) was indicated by conventional (culture methods) and 16S rRNA gene sequence analysis. The overall count of bacteria was a thousand-fold higher than that of fungi. Among the fungal isolates Penicillium spp., Aspergillus spp., and Candida spp. were the most dominant genera. The low recovery of opportunistic pathogenic microorganisms: E. coli, P. stutzeri and C. albicans indicated the low risk of transmission of pathogens harmful to humans by Polish currency.

\section{Acknowledgments}

This research was supported by WEBER SHANDWICK (grant No. BZ-01-0000000000-004-10, ZFIN 00000110, Microbiological Analysis of Banknotes and Coins, 2010).

\section{Literature}

Ahmed S.U., S. Parveen, T. Nasreen and B. Feroza. 2010. Evaluation of the microbial contamination of Bangladesh paper currency notes (Taka) in circulation. Adv. Biol. Res. 4: 266-271.

Clarridge J.E., III. 2004. Impact of $16 \mathrm{~S}$ rRNA gene sequence analysis for identification of bacteria on clinical microbiology and infectious diseases. Clin. Microbiol. Rev. 17: 840-862.

Enemuor S.C., P.I. Victor and O.O. Oguntibeju. 2012. Microbial contamination of currency counting machines and counting room environment in selected commercial banks. Sci. Res. Essays 17: 1501-1511.

Felsenstein J. 1985. Confidence limits on phylogenies: An approach using the bootstrap. Evolution 39: 783-791.

Hall T.A. 1999. BioEdit: a user-friendly biological sequence alignment editor and analysis program for Windows 95/98/NT. Nucl. Acids Symp. Ser. 41: 95-98.

Huber T., G. Faulkner and P. Hugenholtz. 2004. Bellerophon; a program to detect chimeric sequences in multiple sequence alignments. Bioinformatics 20: 2317-2319.

Janda J.M. and S.L. Abbott. 2007. 16S rRNA Gene sequencing for bacterial identification in the diagnostic laboratory: pluses, perils, and pitfalls. J. Clin. Microbiol. 45: 2761-2764.

Jiang X. and M.P. Doyle. 1999. Fate of Escherichia coli O157:H7 and Salmonella enteritidis on currency. J. Food Prot. 62: 805-807.

Kimura M. 1980. A simple method for estimating evolutionary rate of base substitutions through comparative studies of nucleotide sequences. J. Mol. Evol. 16: 111-120.

Khin N.O., P.W. Phyu, M.H. Aung and T. Aye. 1989. Contamination of currency notes with enteric bacterial pathogens. J. Diarrhoeal Dis. Res. 7: 92-94.

Kloos W.E. and T.L. Bannerman. 1999. Staphylococcus and Micrococcus, pp. 264-282. In: Manual of Clinical Microbiology, $7^{\text {th }}$ ed. ASM Press, Washington, D.C.

Kramer A., I. Schwebke and G. Kampf. 2006. How long do nosocomial pathogens persist on inanimate surfaces? A systematic review. BMC Infect. Dis. 6: 1-8.

Kuria J.K., R.G. Wahome, M. Jobalamin and S.M. Kariuki. 2009. Profile of bacteria and fungi on money coins. East Afr. Med. J. 86: 151-155.

Lalucat J., A. Bennasar, R. Bosch, E. García-Valdés and N.J. Palleroni. 2006. Biology of Pseudomonas stutzeri. Microbiol. Mol. Biol. Rev. 70: 510-547.

Madden T.L., R.L. Tatusov and J. Zhang. 1996. Application of network BLAST server. Methods Enzymol. 266: 131-141.

Patel M., J.T. Shackleton and M.M. Coogan. 2006. Effect of antifungal treatment on the prevalence of yeasts in HIV-infected subjects. J. Med. Microbiol. 55: 1279-1284.

Prasai T., K.D. Yami and D.R. Joshi. 2008. Microbial load on paper/ polymer currency and coins. Nepal J. Sci. Technol. 9: 105-109.

Santo C.E., P.V. Morais and G. Grass. 2010. Isolation and characterization of bacteria resistant to metallic copper surfaces. Appl. Environ. Microbiol. 76: 1341-1348.

Tamura K., D. Peterson, N. Peterson, G. Stecher, M. Nei and S. Kumar. 2011. MEGA5: molecular evolutionary genetics analysis using maximum likelihood, evolutionary distance, and maximum parsimony methods. Mol. Biol. Evol. 28: 2731-2739.

Thompson J.D., T.J. Gibson, F. Plewniak, F. Jeanmougin and D.G. Higgins. 1997. The ClustalX windows interface: flexible strategies for multiple sequence alignment aided by quality analysis tools. Nucleic Acids Res. 25: 4876-4882.

Uneke C.J. and O. Ogbu. 2007. Potential for parasite and bacteria transmission by paper currency in Nigeria. J. Environ. Health 69: $54-60$.

Weisburg W.G., S.M. Barns, D.A. Pelletier and D.J. Lane. 1991. $16 \mathrm{~S}$ ribosomal DNA amplification for phylogenetic study. J. Bacteriol. 173: 697-703.

Yazah A.J., J. Yusuf and A.J. Agbo. 2012. Bacterial contaminants of Nigerian currency notes and coins associated with risk factors. Res. J. Med. Sci. 6: 1-6.

Xu J., J.E. Moore and B.C. Millar. 2005. Ribosomal DNA (rDNA) identification of the culturable bacterial flora on monetary coinage from 17 currencies. J. Environ. Health 67: 51-55. 